\title{
VARIATION OF PLASTIC STRAIN RATIOS OF $\alpha$-BRASS SHEET WITH TENSILE STRAIN
}

\author{
HYO-TAE JEONG, SEUNG-HYUN HONG \\ and DONG NYUNG LEE*
}

\author{
Division of Materials Science and Engineering, Seoul National University, \\ Seoul 151-742, Korea
}

(Received in final form 28 September 1997)

\begin{abstract}
A study has been made of the changes in the instantaneous plastic strain ratios along various directions of $\alpha$-brass sheet as a function of tensile strain. The $\alpha$-brass sheet was fabricated by $88 \%$ cold rolling and subsequent annealing at $450^{\circ} \mathrm{C}$ for $1.5 \mathrm{~h}$, which lead to complete recrystallization. The recrystallization texture of the $\alpha$-brass sheet could be approximated by $\{110\}\langle 110\rangle$. The plastic strain ratio along the rolling direction decreased with increasing tensile strain, whereas those along $45^{\circ}, 90^{\circ}$ to the rolling direction were almost independent of tensile strain. The results were in agreement with those calculated using the recrystallization textures based on the Taylor-Bishop-Hill full constraints model.
\end{abstract}

Keywords: $\alpha$-brass; Instantaneous plastic strain ratio; Texture; Tensile strain; Taylor-Bishop-Hill theory; Full constraints model

\section{INTRODUCTION}

Variation of the plastic strain ratio or the Lankford parameter with tensile strain has been reported by many investigators $(\mathrm{Hu}, 1975 \mathrm{a}, \mathrm{b}$; Truzkowski, 1976; Arthey and Hutchinson, 1981; Liu, 1983). In order to understand the phenomenon, it is convenient to classify the plastic strain ratio into the conventional and instantaneous plastic strain ratios.

\footnotetext{
* Corresponding author.
} 
The conventional plastic strain ratio, $R_{\mathrm{e}}$, suggested by Lankford is defined by

$$
R_{\mathrm{e}}=\varepsilon_{\mathrm{w}} / \varepsilon_{\mathrm{t}}=-\varepsilon_{\mathrm{w}} /\left(\varepsilon_{1}+\varepsilon_{\mathrm{w}}\right)
$$

where $\varepsilon_{\mathrm{w}}, \varepsilon_{\mathrm{t}}$ and $\varepsilon_{1}$ are the true plastic strains in the width, thickness and tensile directions, respectively. It is noted that no volume change in plastic deformation is assumed in Eq. (1). The $R_{\mathrm{e}}$ value is usually calculated from the measured $\varepsilon_{\mathrm{w}}$ and $\varepsilon_{1}$ values, and is widely used to evaluate deep drawability of sheet metals.

If the $R_{\mathrm{e}}$ value is independent of tensile strain, the $\varepsilon_{1}-\varepsilon_{\mathrm{w}}$ curve will show a linear relation as shown in Fig. 1, where the slope of line is given by $-R_{\mathrm{e}} /\left(R_{\mathrm{e}}+1\right)$. If there is no error, the line must start from zero point. However, the measured $\varepsilon_{1}-\varepsilon_{\mathrm{w}}$ results often show relations as in Fig. 2. The first case shows a linear relation of $\varepsilon_{1}-\varepsilon_{\mathrm{w}}$ except in the initial stage, whereas the second case shows no linear relation at all. The first case may again be classified into two cases as shown in Fig. 3(a) and (b). For Fig. 3(a) and (b), $R_{\mathrm{e}}$ will behave as shown in Fig. 3(c) and (d) as a function of tensile strain $\varepsilon_{1}$.

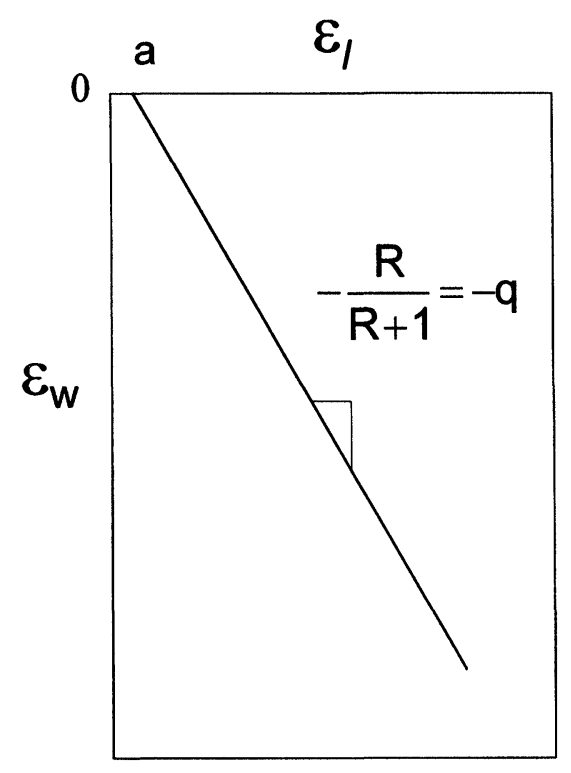

FIGURE 1 Schematic drawing of width strain $\varepsilon_{\mathrm{w}}$ as function of tensile strain $\varepsilon_{1}$ when plastic strain ratio $R$ is independent of strain. 


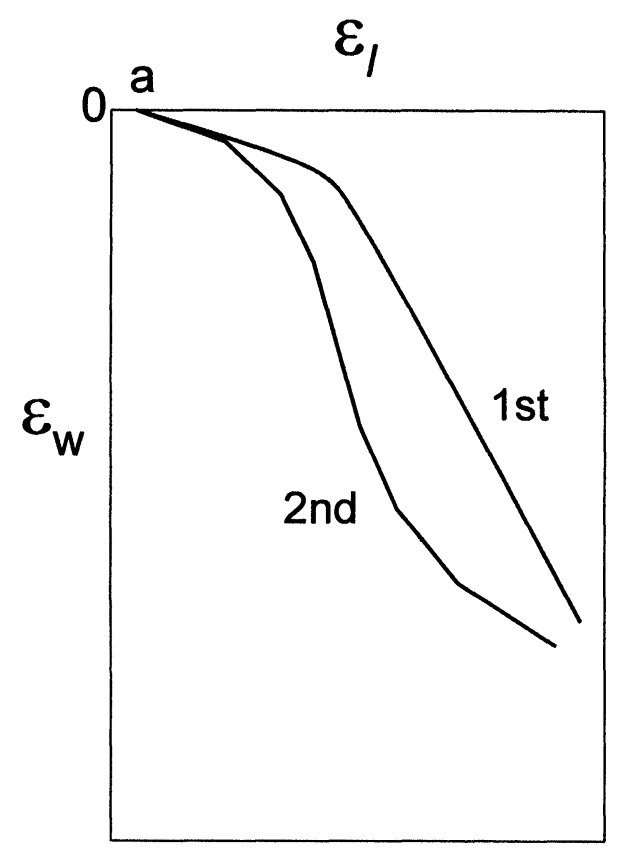

FIGURE 2 Schematic drawing of width strain $\varepsilon_{\mathrm{w}}$ as function of tensile strain $\varepsilon_{1}$ when plastic strain ratio $R$ varies with strain.

If the plastic strain ratio is defined by the instantaneous slope of the $\varepsilon_{1}-\varepsilon_{\mathrm{w}}$ curve, the results in Fig. 3(a) and (b) will give rise to the curves $R_{\mathrm{i}}$ in Fig. 3(c) and (d). For the second curve in Fig. 2, $R_{\mathrm{e}}$ and $R_{\mathrm{i}}$ are defined in Fig. 4(Welch et al., 1983; Lake et al., 1988). $R_{\mathrm{i}}$ is called the instantaneous plastic strain ratio and is expressed as

$$
R_{\mathrm{i}}=\mathrm{d} \varepsilon_{\mathrm{w}} / \mathrm{d} \varepsilon_{\mathrm{t}} .
$$

The instantaneous plastic strain ratio corresponds to the quantity that is usually calculated from the texture.

The objective of this article is to measure the instantaneous plastic strain ratio of $\alpha$-brass sheet as a function of tensile strain and to discuss the results based on its textures.

\section{EXPERIMENTAL METHOD}

The $0.8 \mathrm{~mm}$ thick $\alpha$-brass sheet $(\mathrm{Cu}-28 \% \mathrm{Zn})$ was made by $88 \%$ cold rolling at room temperature, followed by annealing at $450^{\circ} \mathrm{C}$ for $1.5 \mathrm{~h}$. 


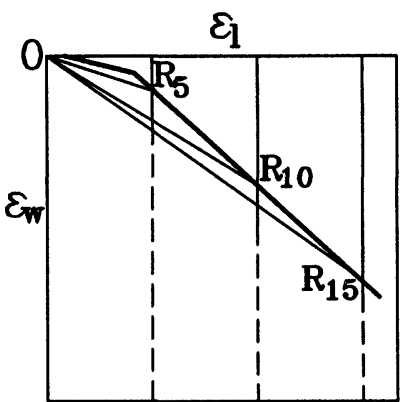

(a)

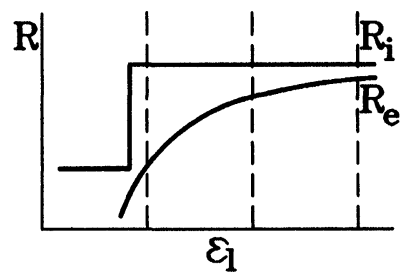

(c)

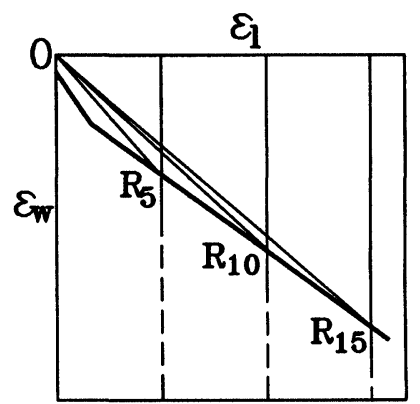

(b)

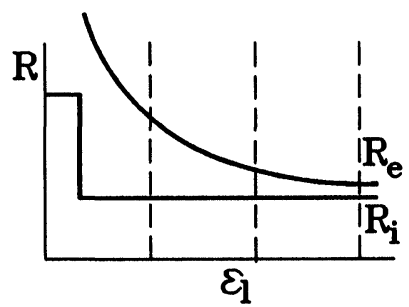

(d)

FIGURE 3 Difference between conventional plastic strain ratio $R_{\mathrm{e}}$ and instantaneous plastic strain ratio $R_{\mathrm{i}}$.

The tensile specimens of ASTM E8 subsize were prepared by milling the $\alpha$-brass sheets.

The width and longitudinal displacements during tensile testing were instantaneously measured using two extensometers. The longitudinal displacement was measured up to $10 \mathrm{~mm}$ at intervals of $4.8 \mu \mathrm{m}$, while the width displacement was measured up to $1.5 \mathrm{~mm}$ at intervals of $0.69 \mu \mathrm{m}$, which is equivalent to about 1000 data at a tensile strain of 0.2 . The measured displacements were used to calculate strains, from which the plastic strains were obtained by subtracting the elastic strains. The elastic strains were calculated using the following equation:

$$
\varepsilon_{\mathrm{w}}^{\mathrm{e}}=-\nu \varepsilon_{1}^{\mathrm{e}}=\frac{\nu}{E} \sigma\left(\varepsilon_{1}\right)
$$

where superscript e represents elastic property, and $\nu$ is Poisson's ratio. Equation (3) holds for isotropic elastic materials. Therefore, the 


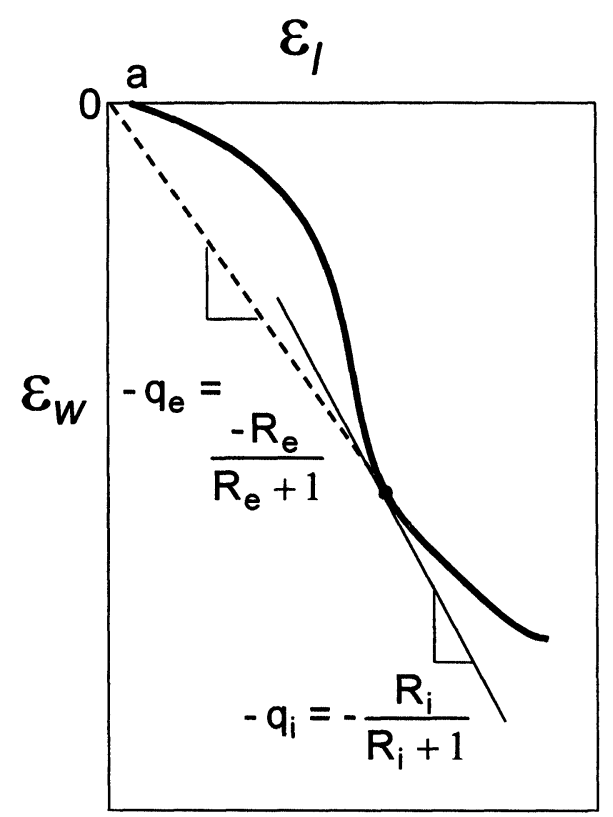

FIGURE 4 Definition of conventional plastic strain ratio $R_{\mathrm{e}}$ and instantaneous plastic strain ratio $R_{\mathrm{i}}$, where $-q_{\mathrm{e}}$ and $-q_{\mathrm{i}}$ are slopes of dashed and sold lines.

effect of elastic anisotropy on the plastic strains was neglected. Young's modulus and Poisson's ratio used are $115 \mathrm{GPa}$ and 0.343 , respectively.

In order to reduce the noise of the measured data, an instantaneous plastic strain ratio was calculated from the slope of a quadratic equation best fitting 50 data obtained from a tensile strain range of $\pm 0.5 \%$.

The textures of the annealed specimens were measured up to the reflection angle of $80^{\circ}$ using a Schultz pole figure device. Incomplete pole figures of (111), (200) and (220) for the $\alpha$-brass specimen were used to calculate ODFs using the series expansion method with $L_{\max }=22$ (Bunge, 1982).

\section{RESULTS AND DISCUSSION}

Figure 5 shows the measured width strains and the $R_{\mathrm{i}}$ values along $0^{\circ}, 45^{\circ}$ and $90^{\circ}$ to the rolling direction of $\alpha$-brass specimens as a function of 
(a) El
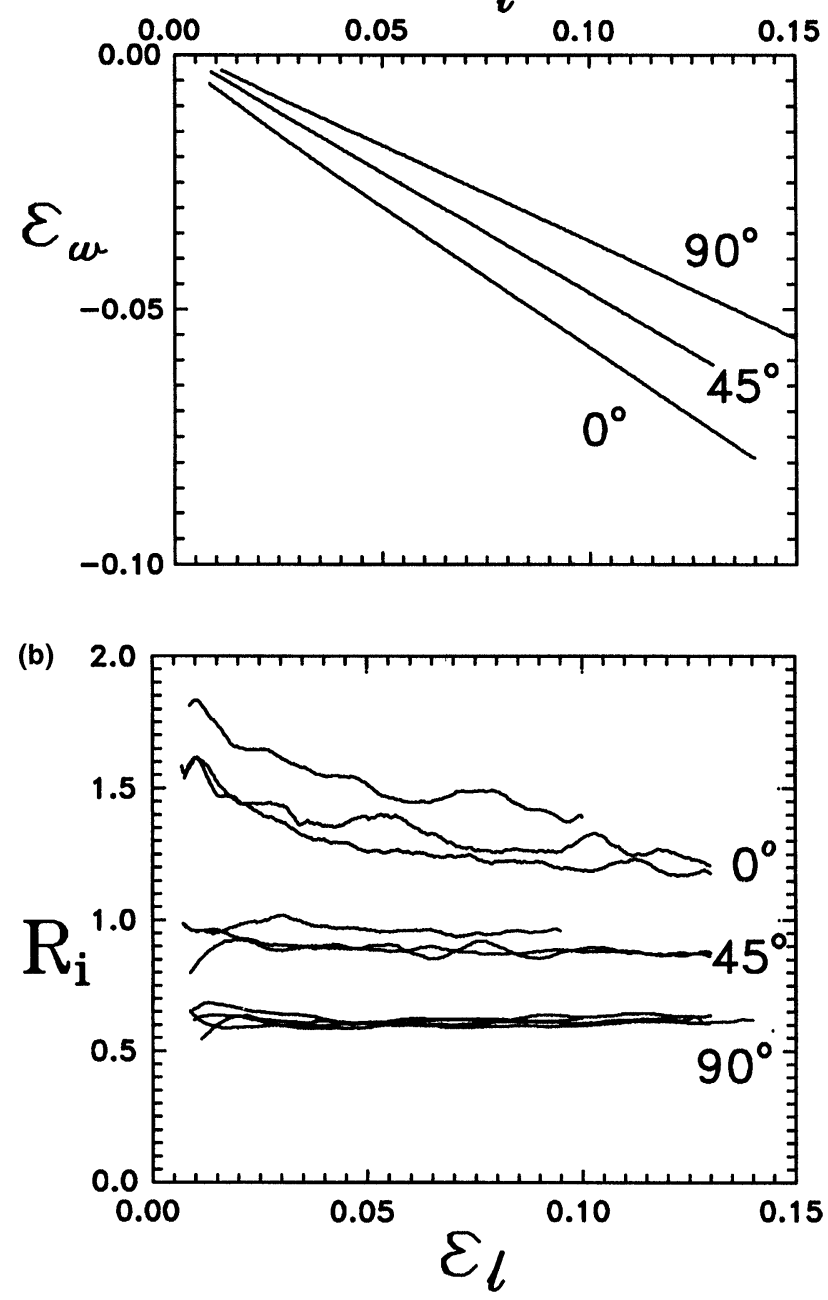

FIGURE 5 Measured (a) width strain $\varepsilon_{\mathrm{w}}$ and (b) instantaneous plastic strain ratio $R_{\mathrm{i}}$ of annealed $\alpha$-brass sheet as function of tensile strain $\varepsilon_{1}$.

tensile strain. The $R_{\mathrm{i}}$ values of $45^{\circ}$ and $90^{\circ}$ specimens are independent of the tensile strain, whereas the $R_{\mathrm{i}}$ values of $0^{\circ}$ specimen decreases with increasing tensile strain.

Figure 6 shows the measured (111) and (200) pole figures of the annealed specimen. Figure 7 shows the measured (111) and (200) pole 


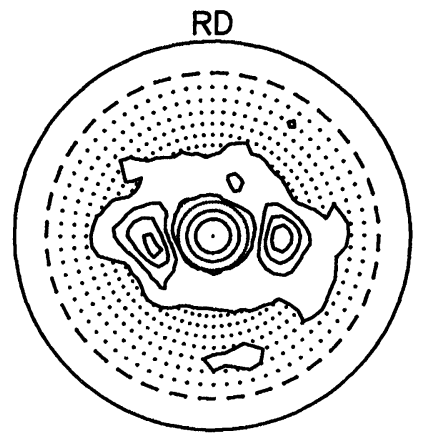

Max. $\quad 2.8$

Contour $1.0 \quad 1.5 \quad 2.02 .5$

(111)

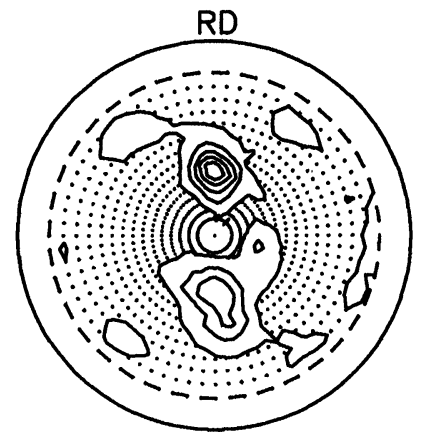

Max. $\quad 3.5$

Contour $1.01 .5 \quad 2.02 .5 \quad 3.0$

(200)

FIGURE 6 Measured (111) and (200) pole figures of annealed $\alpha$-brass sheet.

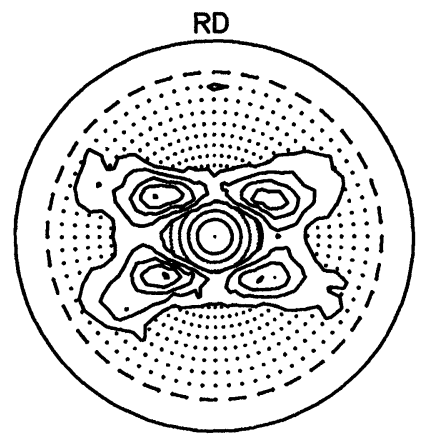

Max.

Contour $1.0 \quad 1.5 \quad 2.0 \quad 2.5 \quad 3.0$

(111)

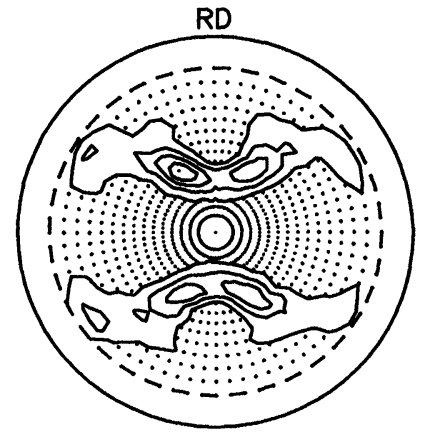

Max. $\quad 3.0$

Contour $1.01 .5 \quad 2.0 \quad 2.5 \quad 3.0$

(200)

FIGURE 7 Measured (111) and (200) pole figures of $\alpha$-brass sheet tensile deformed by $33 \%$.

figures of the specimen tensile deformed by $33 \%$ along the rolling direction. The ODFs calculated using the pole figures in Figs. 6 and 7 are shown in Fig. 8(a) and (b), respectively. The texture of the annealed specimen may be approximated by the $\{110\}\langle 110\rangle$ orientation and the texture of the tensile deformed specimen may be approximated by the $\{110\}\langle 111\rangle$ orientation. 


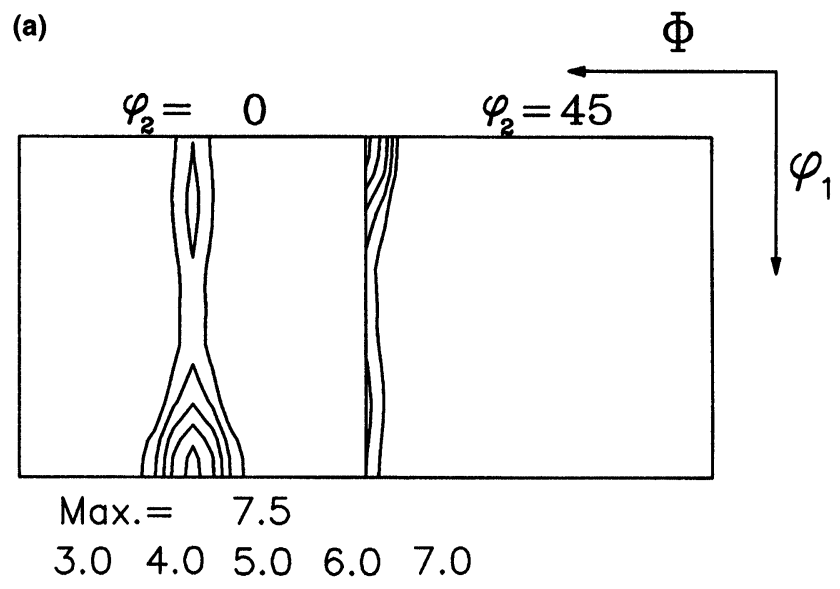

(b)

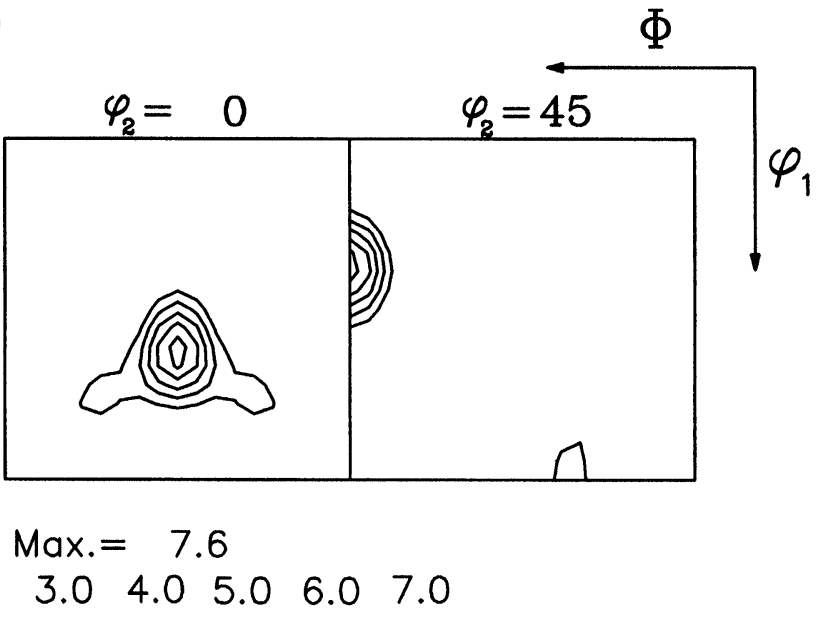

FIGURE 8 ODFs at $\varphi_{2}=0$ and $45^{\circ}$ sections of $\alpha$-brass sheet (a) annealed at $450^{\circ} \mathrm{C}$ for $1.5 \mathrm{~h}$ after $88 \%$ rolling and (b) tensile deformed by $33 \%$.

The $R_{\mathrm{i}}$ values of the annealed and tensile deformed specimens were calculated as a function of angle to the rolling direction using Bunge's method (Bunge, 1982) based on the measured textures (Fig. 9). The calculated $R_{\mathrm{i}}$ values of the annealed specimen along $0^{\circ}, 45^{\circ}$ and $90^{\circ}$ to the rolling direction are $1.60,0.86$ and 0.46 , respectively, which are in good agreement with the measured $R_{\mathrm{i}}$ values at the small tensile strain (Fig. 5(b)). The calculated $R_{\mathrm{i}}$ values of the tensile deformed specimens 




FIGURE 9 Instantaneous plastic strain ratio calculated using Bunge's method as function of angle to rolling direction for annealed and tensile deformed $\alpha$-brass sheet.

along $45^{\circ}$ and $90^{\circ}$ to the rolling direction are similar to those of the annealed specimens, whereas the $R_{\mathrm{i}}$ value along the rolling direction decreases drastically. This decrease of $R_{\mathrm{i}}$ value along the rolling direction was attributed to the observed texture change from $\{110\}\langle 110\rangle$ to $\{110\}\langle 111\rangle$.

For qualitative understanding of the $R_{\mathrm{i}}$ results, the development of texture was calculated as a function of tensile strain, from which the $R_{\mathrm{i}}$ was calculated. The annealing texture of $\alpha$-brass sheet was expressed as the Gaussian function of $\{110\}\langle 110\rangle$ orientation with a half width of $30^{\circ}$ and the calculated pole figures and ODF are shown in Figs. 10 and 11(a), respectively. Therefore, the annealing texture of $45^{\circ}$ and $90^{\circ}$ specimens was expressed as Gaussian functions of $\{110\}\langle 11 \sqrt{2}\rangle$ and $\{110\}\langle 001\rangle$ orientations with a half width of $30^{\circ}$.

The tensile deformation textures were calculated using the TaylorBishop-Hill full constraints model, in which the slip systems were 


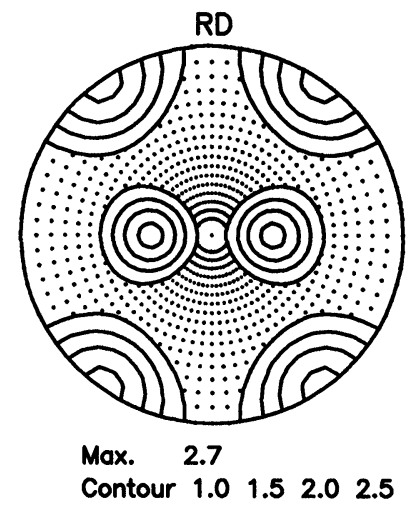

(111)

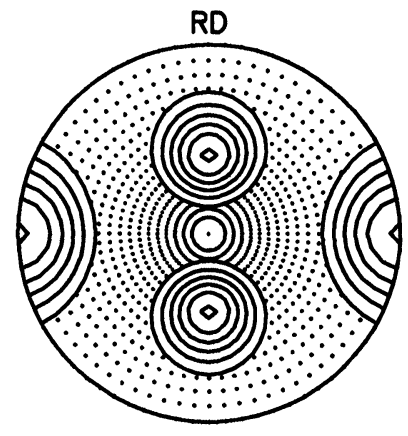

Max. $\quad 3.6$

Contour $1.01 .5 \quad 2.02 .53 .03 .5$

(200)

FIGURE 10 (111) and (200) pole figures of $\{110\}\langle 110\rangle$ texture expressed as Gaussian function with $L_{\max }=22$ and $\omega_{\mathrm{o}}=30^{\circ}$.

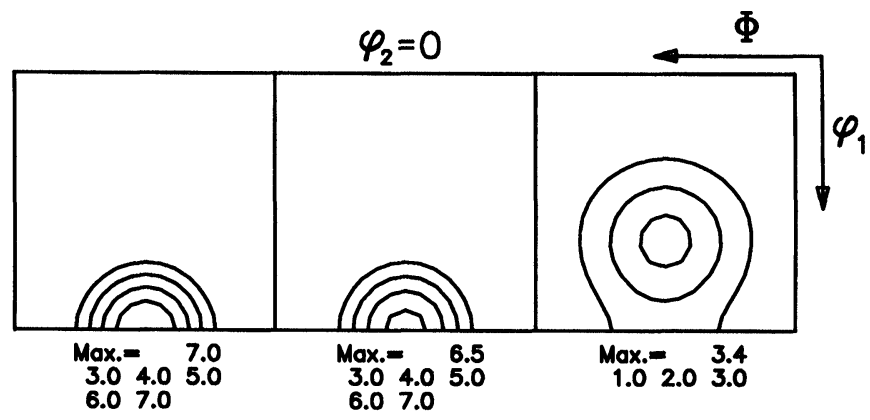

(a)

(b)

(c)

FIGURE 11 ODFs at $\varphi_{2}=0^{\circ}$ section of $\alpha$-brass sheet tensile deformed along the rolling direction, which were calculated using Taylor-Bishop-Hill full constraint model. (a) $\varepsilon_{1}=0.0$, (b) $\varepsilon_{1}=0.4$, (c) $\varepsilon_{1}=1.5$.

selected using the random choice of linear programming method (Van Houtte, 1981). The calculated deformation textures are shown in Figs. $11-13$. The $\{110\}\langle 110\rangle$ and $\{110\}\langle 11 \sqrt{2}\rangle$ orientation of $0^{\circ}$ and $45^{\circ}$ specimens are rotated to $\{110\}\langle 111\rangle$ orientation during tensile deformation, while the $\{110\}\langle 001\rangle$ orientation of $90^{\circ}$ specimen is stable. The calculated annealing and deformation textures were used to calculate the $R_{\mathrm{i}}$ values using Bunge's method. The calculated results are shown in Fig. 14. It can be seen that the $0^{\circ}$ specimen of $\{110\}\langle 110\rangle$ orientation yields $R_{\mathrm{i}}$ values which strongly decrease with increasing tensile strains, whereas 


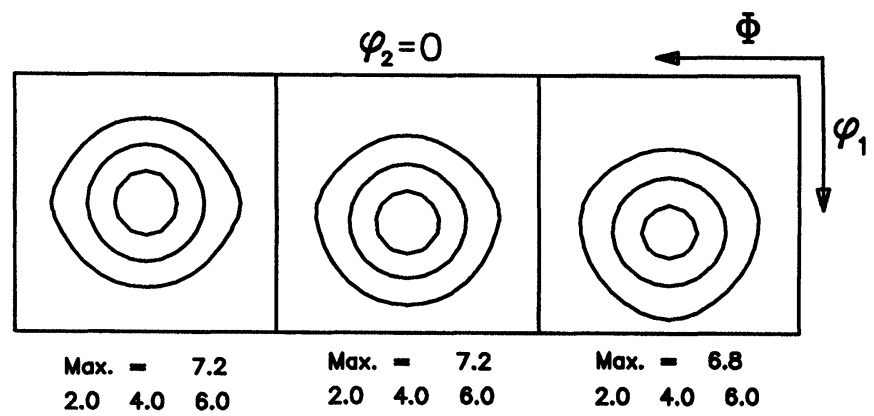

(a)

(b)

(c)

FIGURE 12 ODFs at $\varphi_{2}=0^{\circ}$ section of $\alpha$-brass sheet tensile deformed along $45^{\circ}$ to rolling direction, which were calculated using Taylor-Bishop-Hill full constraint model. (a) $\varepsilon_{1}=0.0$, (b) $\varepsilon_{1}=0.4$, (c) $\varepsilon_{1}=1.5$.

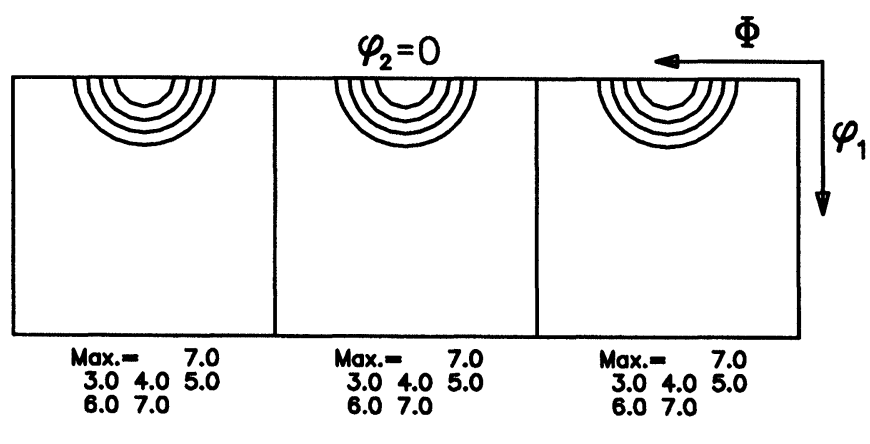

(a)

(b)

(c)

FIGURE 13 ODFs at $\varphi_{2}=0^{\circ}$ section of $\alpha$-brass sheet tensile deformed along $90^{\circ}$ to rolling direction, which were calculated using Taylor-Bishop-Hill full constraint model. (a) $\varepsilon_{1}=0.0$, (b) $\varepsilon_{1}=0.4$, (c) $\varepsilon_{1}=1.5$.

the $R$ values of the $45^{\circ}$ and $90^{\circ}$ specimens are virtually independent of the tensile strain.

These results are in qualitative agreement with the measured $R_{\mathrm{i}}$ values. Therefore, the variations of $R_{\mathrm{i}}$ with tensile strain can be attributed to the texture change in $\alpha$-brass sheets during tensile deformation.

\section{CONCLUSION}

The instantaneous plastic strain ratios and textures of $\alpha$-brass sheets were measured as functions of tensile strain and angle to the rolling 


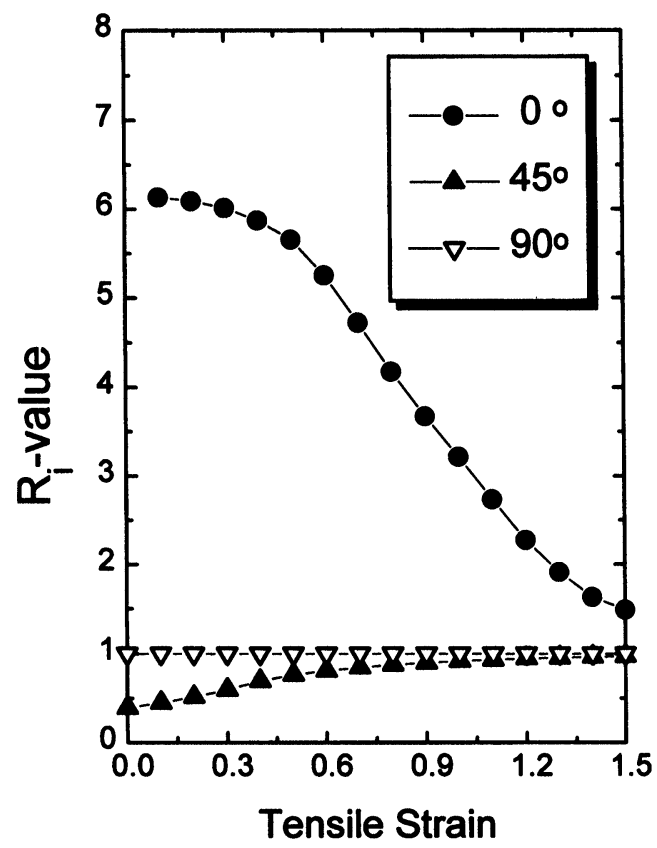

FIGURE 14 Variation of instantaneous plastic strain ratio calculated based on development of deformation texture using Bunge's method as function of tensile strain for $\alpha$-brass sheet.

direction. The recrystallization texture of $\alpha$-brass sheet rolled by $88 \%$ and annealed at $450^{\circ} \mathrm{C}$ for $1.5 \mathrm{~h}$ was approximated by $\{110\}\langle 110\rangle$, which changed to the texture approximated by $\{110\}\langle 111\rangle$ after a tensile strain of $33 \%$. The $R_{\mathrm{i}}$ values of specimens along $45^{\circ}$ and $90^{\circ}$ to the rolling direction were almost independent of tensile strain, whereas that of the $0^{\circ}$ specimen decreased with increasing tensile strain. The $R_{\mathrm{i}}$ results are attributed to the texture variation during tensile deformation.

\section{Acknowledgments}

This study has been supported by Korea Science and Engineering Foundation through RETCAM, Seoul National University.

\section{References}

Arthey, R.P. and Hutchinson, W.B. (1981) Metall. Trans. A, 12, 1817.

Bunge, H.J. (1982) “Texture Analysis in Materials Science” Butterworths, London, p. 330 
Hu, H. (1975a). Metall. Trans. A, 6, 2307.

Hu, H. (1975b). Metall. Trans. A, 6, 945.

Lake, J.S.H., Willis, D.J. and Fleming, H.G. (1988). Metall. Trans. A, 19, 2805.

Liu, Y.C. (1983). Metall. Trans. A, 14, 1199.

Truzkowski, W. (1976). Metall. Trans. A, 7, 327.

Van Houtte, P. (1981). ICOTOM 6, Tokyo, 1, 428.

Welch, P.I., Ratke, L. and Bunge, H.-J. (1983). Z. Metallkde., 74(4), 233. 